\title{
On Nonlinear Evolution of Axisymmetric Nuclear Surface
}

\author{
V.G. Kartavenko ${ }^{1,2}$, K.A. Gridnev ${ }^{3}$ and W.Greiner ${ }^{2}$ \\ ${ }^{1}$ Bogoliubov Laboratory of Theoretical Physics, Joint Institute for Nuclear Research, \\ Dubna, Moscow District, 141980, Russia \\ ${ }^{2}$ Institut für Theoretische Physik der J. W. Goethe Universität \\ D-60054 Frankfurt am Main, Germany \\ 3 Department of Nuclear Physics, Institute of Physics, \\ St.Petersburg State University, 198904, Russia
}

(November 2000)

\begin{abstract}
We consider an uniformly charged incompressible nuclear fluid bounded by a closed surface. It is shown that an evolution of an axisymmetric surface $\Gamma(\boldsymbol{r}, t) \equiv \sigma-\Sigma(z, t)=0, \quad \boldsymbol{r}=(\sigma, \phi, z)$ can be approximately reduced to a motion of a curve in the $(\sigma, z)$-plane. A nonlinear integro-diffrerential equation for the contour $\Sigma(z, t)$ is derived. It is pointed on a direct correspondence between $\Sigma(z, t)$ and a local curvature, that gives possibility to use methods of differential geometry to analyze an evolution of an axisymmetric nuclear surface.
\end{abstract}

\section{MOTIVATION}

Nonlinear dynamics of a nuclear surface is an object of special interest due to the following reasons. First, nuclear density falls considerably in the surface region, where the density fluctuations and clustering may be important. Second, different types of instability may be developed in the surface region and lead to fragmentation processes at low (fission, nucleon transfer) and high (multifragmentation, break-up etc.) energies. Finally, the liquid drop concept [1] for over a century are intensively used in macro- [2] and micro-physics [3]. Nonlinear dynamics of shapes in any complicated systems enevitably leads to mathematical problem of describing global geometric quantities such as surface and enclosed volume in different dimensions (polymers, cell membranes, 3D droplets).

An application of a soliton concept to nonlinear nuclear hydrodynamics has given yet new possibilities in this field (See e.g. review [4] and [5] 8] for the recent refs.). However any extension of nonlinear dynamics from $1+1$ to $2+1$ and $3+1$ dimensions meets principal difficulties. The crucial point is to reduce the dimension of a problem. In paper [9] we considered the simplest two-dimensional nonlinear liquid objects. It was shown that 2D pure vortical motion of inviscid nuclear liquid can be reduced to 1D evolution of the contour bounding this drop. In the next paper 10] the extension to semi-3D geometry was done. The equations of motion describing localized vortex on a spherical nuclear surface - a bounded region of constant vorticity, surrounded by irrotational flux - were reduced to 1D nonlinear evolution of the boundary.

In this short report we consider an uniformly charged incompressible nuclear 3D fluid bounded by a closed surface. It is shown that evolution of an axisymmetric surface $\Gamma(\boldsymbol{r}, t) \equiv \sigma-\Sigma(z, t)=0, \quad \boldsymbol{r}=(\sigma, \phi, z)$ can be approximately reduced to a motion of a curve in the $(\sigma, z)$-plane.

\section{FRAMEWORK}

The evolution of one-body Wigner phase-space distribution function is analyzed instead of a full many-body wave function. Integrating the kinetic equation

$$
\frac{\partial f}{\partial t}+\frac{\boldsymbol{p}}{m} \cdot \frac{\partial f}{\partial \boldsymbol{r}}-\frac{\partial V}{\partial \boldsymbol{r}} \cdot \frac{\partial f}{\partial \boldsymbol{p}}=I_{r e l}, \quad H_{W}=\frac{p^{2}}{2 m}+V(\boldsymbol{r})
$$

over the momentum space with different polynomial weighting functions of the $\boldsymbol{p}$-variable one comes to an infinite chain of equations for local collective observables including the density, collective velocity, pressure and an infinite set of tensorial functions of the time and space coordinates, which are defined as moments of the distribution function in the momentum space:

- the particle $n(\boldsymbol{r}, t) \equiv g \int d \boldsymbol{p} f(\boldsymbol{r}, \boldsymbol{p}, t)$, and the mass $\rho(\boldsymbol{r}, t)=m n(\boldsymbol{r}, t)$ densities, where we consider a proton and neutron as different state of identical particle (the spin-isospin degeneration $g=4$ ).

- the collective current and velocity of nuclear matter $\rho(\boldsymbol{r}, t) \boldsymbol{u}(\boldsymbol{r}, t)=g \int d \boldsymbol{p} \boldsymbol{p} f(\boldsymbol{r}, \boldsymbol{p}, t)$ 
- the pressure tensor $P_{i j}(\boldsymbol{r}, t)=(g / m) \int d \boldsymbol{p} q_{i} q_{j} f(\boldsymbol{r}, \boldsymbol{p}, t), \quad \boldsymbol{q}=\boldsymbol{p}-m \boldsymbol{u}$

- the energy and momentum transfer tensors of a different order

$$
P_{i j k}(\boldsymbol{r}, t)=\frac{g}{m^{2}} \int d \boldsymbol{p} q_{i} q_{j} q_{k} f(\boldsymbol{r}, \boldsymbol{p}, t), \quad \underbrace{P_{i j . . k}}_{n}(\boldsymbol{r}, t)=\frac{g}{m^{n-1}} \int d \boldsymbol{p} \underbrace{q_{i} q_{j} . . q_{k}}_{n} f(\boldsymbol{r}, \boldsymbol{p}, t),
$$

- and the integrals related to relaxation terms

$$
\int d \boldsymbol{p} I_{r e l}=0, \quad \int d \boldsymbol{p} \boldsymbol{p} I_{r e l}=0, \quad R_{i j} \equiv \frac{g}{m} \int d \boldsymbol{p} q_{i} q_{j} I_{r e l}, \quad \ldots
$$

Truncating this chain one arrives at the "fluid dynamical" level of description of nuclear processes.

$$
\begin{gathered}
\frac{\partial \rho}{\partial t}+\sum_{k} \frac{\partial}{\partial x_{k}}\left(u_{k} \rho\right)=0 \\
\rho \frac{D u_{i}}{D t}+\sum_{k} \frac{\partial P_{i k}}{\partial x_{k}}+\frac{\rho}{m} \frac{\partial V}{\partial x_{i}}+\rho\left(\Omega_{i} \sum_{k} \Omega_{k} x_{k}-\Omega^{2} x_{i}\right) \\
+\rho \sum_{s, j} \varepsilon_{i s j}\left(2 \Omega_{s} u_{j}+\frac{d \Omega_{s}}{d t} x_{j}\right)=0 \\
\frac{D P_{i j}}{D t}+\sum_{k}\left(P_{i k} \frac{\partial u_{j}}{\partial x_{k}}+P_{j k} \frac{\partial u_{i}}{\partial x_{k}}+P_{i j} \frac{\partial u_{k}}{\partial x_{k}}\right) \\
+2 \sum_{s, m} \Omega_{m}\left(\varepsilon_{j m s} P_{i s}+\varepsilon_{i m s} P_{j s}\right)+\sum_{k} \frac{\partial}{\partial x_{k}} P_{i j k}=R_{i j}
\end{gathered}
$$

where the usual notation $\frac{D}{D t} \equiv \frac{\partial}{\partial t}+\sum_{k} u_{k} \frac{\partial}{\partial x_{k}}$ is introduced for the operator giving the material derivative, or rate of change at a point moving with the fluid locally. The hydrodynamical set of Eqs. (146) describes an evolution in a rotating nuclear system. The linear transformation $x_{i}=\sum_{j=1}^{3} T_{i j} X_{j}$ relates the coordinates of a point, $\left(X_{1}, X_{2}, X_{3}\right)$, in an inertial frame, and, $\left(x_{1}, x_{2}, x_{3}\right.$ in a moving frame of reference with a common origin. The orientation of a moving frame, with respect to an inertial frame, will be assumed to be time dependent, $T_{i j}(t)$, representing an orthogonal transformation, and the vector

$$
\Omega_{i}=\frac{1}{2} \sum_{j, k, m} \varepsilon_{i j k}(d T / d t)_{j m} T_{m k}^{+}
$$

represents a general time-dependent rotation.

Let us restrict ourselves with simplest possible motion of nuclear fluid. Consider an uniformly charged (with a total charge $Z e$ ) incompressible nuclear "fluid" confined to a volume $V$ bounded by a closed surface $S$, obeying to the equation $\Gamma(\boldsymbol{r}, t) \equiv \sigma-\Sigma(z, t)=0$, where $(\sigma, \phi, z)$ are cylindrical coordinates of a point. The geometry places the origin at the center-of-mass $\left(\int d \boldsymbol{r} \rho(\boldsymbol{r}, t) \boldsymbol{u}(\boldsymbol{r}, t)=0\right)$. The mean-field potential and related tensors can be decomposed into nuclear and Coulomb terms $V(\boldsymbol{x}, t)=V_{\text {nucl }}(\boldsymbol{x}, t)+V_{\text {coul }}(\boldsymbol{x}, t)$. A nuclear potential can be derived as the first variation of the short-range interaction functional (as usual effective density dependent Skyrme forces) on density $V_{n u c l}(\boldsymbol{x}, t) \equiv \delta \mathcal{E}[n] / \delta n$, This gives a nuclear potential as the function of the density $n(\boldsymbol{r}, t)=n_{0} \eta(\boldsymbol{r}, t)$. The incompressibility of the nuclear matter implies $n_{0}=\left(2 p_{F}^{3}\right) /\left(3 \pi \hbar^{3}\right)$ where $n_{0}, p_{F}$ being respectively, the density of nuclear matter and the Fermi momentum. Or, keeping in mind that an effective mean-field potential approximately constant inside the nuclei and has a sharp coordinate dependence in the surface region, to replace it by the surface term [1]] $\gamma_{s}$ div $\hat{\boldsymbol{n}}$, where $\hat{\boldsymbol{n}}=\nabla \Gamma /|\nabla \Gamma|$ is the unit outward normal on $S$, and the surface tension $\gamma_{s}$ is related to mass formula coefficient via $b_{s}=4 \pi r_{0}^{2} \gamma_{s} \sim 22 \mathrm{MeV}$. In the both cases the contour $\Sigma(z, t)$ defines completely a nuclear potential on a nuclear surface. 


$$
\begin{array}{r}
V_{\text {coul }}(\boldsymbol{x}, t)=\left(\frac{Z}{A} e\right)^{2} n_{0} \int d \boldsymbol{x}^{\prime} \frac{\eta\left(\boldsymbol{x}^{\prime}, t\right)}{\left|\boldsymbol{x}-\boldsymbol{x}^{\prime}\right|}= \\
\left(\frac{Z}{A} e\right)^{2} n_{0} \int_{z_{\text {min }}}^{z_{\text {max }}} d z^{\prime} \int_{0}^{\Sigma\left(z^{\prime}, t\right)} d \sigma^{\prime} \sigma^{\prime} I\left(\sigma, \sigma^{\prime}, z^{\prime}\right), \\
I\left(\sigma, \sigma^{\prime}, z^{\prime}\right)=\int_{0}^{2 \pi} d \phi \frac{1}{\sqrt{\sigma^{2}+\sigma^{\prime 2}-2 \sigma \sigma^{\prime} \cos \phi}},
\end{array}
$$

Concerning pressure tensors, we will not solve the kinetic equation (11), but will use that, this simplest picture can be reproduced by assuming the following factorised form of the distribution functions $f(\boldsymbol{r}, \boldsymbol{p}, t)$

$$
f^{0}(\boldsymbol{r}, \boldsymbol{p})=(2 \pi \hbar)^{-3} \eta^{0}(\boldsymbol{r}) \phi^{0}(\boldsymbol{p}) \quad f(\boldsymbol{r}, \boldsymbol{p}, t)=(2 \pi \hbar)^{-3} \eta(\boldsymbol{r}, t) \phi(\boldsymbol{r}, \boldsymbol{p}, t),
$$

where $\eta(\boldsymbol{r}, t) \equiv \theta(\Gamma(\boldsymbol{r}, t)) \quad\left(\eta^{0}(\boldsymbol{r}) \equiv \theta\left(\Gamma^{0}(\boldsymbol{r})\right)\right)$ is the step-function which is equal to one inside the surface $\Gamma(\boldsymbol{r}, t)$ $\left(\Gamma^{0}(\boldsymbol{r})\right)$ and zero outside it. The distribution function $f^{0}(\boldsymbol{r}, \boldsymbol{p})$ describes the equilibrium spherical symmetrical state $\left.\left(\Gamma^{0}(\boldsymbol{r})\right) \equiv \sigma-\sqrt{R_{0}^{2}-z^{2}}\right)$, where $R_{0}$ is the radius of a sphere of an equivalent volume $\left(4 R_{0}^{3}=3 \int d z \Sigma(z, t)^{2}\right)$. The distribution function $f(\boldsymbol{r}, \boldsymbol{p}, t)$ corresponds to the dynamical picture. Both distribution function describe a homogenous distribution of a nuclear matter within the volume bounded by a sharp surface. The momentumdependent part of the distribution functions could be parametrized as follows :

$$
\phi^{0}(\boldsymbol{p})=\theta\left(p_{F}^{2}-p^{2}\right), \quad \phi(\boldsymbol{r}, \boldsymbol{p}, t)=\theta\left(p_{F}^{2}-\sum_{i, j}\left(\delta_{i j}+\alpha_{i j}(t)\right) q_{i} q_{j}\right) .
$$

The forms chosen for the momentum-dependent part of $f(\boldsymbol{r}, \boldsymbol{p}, t),\left(f^{0}(\boldsymbol{r}, \boldsymbol{p}, t)\right)$, insures that it yields the current density with the collective velocity $\boldsymbol{u}(\boldsymbol{r}, t)\left(\boldsymbol{u}^{0}=0\right)$. Of course, symmetry of the problem is defined by the symmetry of the all effective forces and an initial state. In a fluid at rest, only normal stresses are exerted, the normal stress is independent of the direction of the normal to the surface element across which it acts, and the equilibrium pressure tensor has the spherically symmetrical form via symmetry of the equilibrium distribution function $P_{i j}^{0}(\boldsymbol{r}, t)=(2 / 5) \epsilon_{F} \eta^{0}(\boldsymbol{r}) n_{0} \delta_{i j}$. There no reason to expect these results to be valid for a fluid in motion, the tangential stresses are non-zero, in general, and the normal component of the stress acting across a surface element depends on the direction of the normal to the element. The simple notion of a pressure acting equally in all directions is lost in most cases of a fluid in motion. A deformations in the cartesian space define deformation in the momentum space and vice versa. We consider the only motion with $\boldsymbol{\Omega}=0$ and will assume the diagonal tensor $\alpha_{i j}$. The dynamical pressure tensors are $P_{i j}(\boldsymbol{r}, t)=(2 / 5)\left(1+\alpha_{i i}\right)^{-1} \epsilon_{F} n_{0} \eta(\boldsymbol{r}, t) \delta_{i j}$ These equations show that the introduction of the functions $\alpha_{i i}(t)$ into the expression for $\phi(\boldsymbol{r}, \boldsymbol{p}, t)$ accounts for the deformation of the Fermi-surface.

The main hydrodynamical set of equations can be recast in the following form in the cylindrical coordinates

$$
\begin{aligned}
& \frac{\partial u_{\sigma}}{\partial t}+u_{\sigma} \frac{\partial u_{\sigma}}{\partial \sigma}+u_{z} \frac{\partial u_{\sigma}}{\partial z}=F_{\sigma} \\
& \frac{\partial u_{z}}{\partial t}+u_{\sigma} \frac{\partial u_{z}}{\partial \sigma}+u_{z} \frac{\partial u_{z}}{\partial z}=F_{z} \\
& \frac{\partial u_{\sigma}}{\partial \sigma}+\frac{\partial u_{z}}{\partial z}+\frac{u_{\sigma}}{\sigma}=0
\end{aligned}
$$

where $u_{\sigma}, u_{z}\left(F_{\sigma}, F_{z}\right)$ are the projections of the velocity (forces) along the axis $\sigma$ and $z$ respectively.

The boundary conditions are

$$
\hat{\boldsymbol{n}} \Xi \hat{\boldsymbol{n}}=-\gamma_{s}\left(\frac{1}{C_{1}}+\frac{1}{C_{2}}\right), \quad \hat{\boldsymbol{n}} \Xi \hat{\boldsymbol{t}}=0 .
$$

Here $\Xi$ is the stress tensor, which can be calculated using all the formulas above for the pressure tensor and the mean-field potentials. $C_{1}$ and $C_{2}$ are the principal radii of curvature. The direct evaluation gives

$$
\begin{gathered}
\frac{1}{C_{1}}+\frac{1}{C_{2}}=\frac{1}{\Sigma\left(1+\Sigma_{z}^{2}\right)^{1 / 2}}-\frac{\Sigma_{z z}}{\left(1+\Sigma_{z}^{2}\right)^{3 / 2}}, \quad \Sigma_{z} \equiv \frac{\partial \Sigma}{\partial z}, \quad \Sigma_{z z} \equiv \frac{\partial^{2} \Sigma}{\partial z^{2}} \\
\hat{\boldsymbol{n}}=\frac{\hat{e}_{\sigma}-\Sigma_{z} \hat{e}_{z}}{\sqrt{1+\Sigma_{z}^{2}}}, \quad \hat{\boldsymbol{t}}=\frac{\Sigma_{z} \hat{e}_{\sigma}+\hat{e}_{z}}{\sqrt{1+\Sigma_{z}^{2}}} \quad \hat{e}_{\sigma}=\frac{\hat{\boldsymbol{n}}+\Sigma_{z} \hat{\boldsymbol{t}}}{\sqrt{1+\Sigma_{z}^{2}}}, \quad \hat{e}_{z}=\frac{-\Sigma_{z} \hat{\boldsymbol{n}}+\hat{\boldsymbol{t}}}{\sqrt{1+\Sigma_{z}^{2}}},
\end{gathered}
$$


The final integro-differential equation of motion for a contour accumulates the coupled set of equations (10,12)

$$
\frac{D \Sigma(z, t)}{D t}=\frac{\partial \Sigma(z, t)}{\partial t}+u_{z}(\Sigma, z, t) \frac{\partial \Sigma(z, t)}{\partial z}=u_{\sigma}(\Sigma, z, t)
$$

The evolution of the surface $\Gamma(\sigma, z, t)$ as a motion of a $2 \mathrm{D}$ space curve in the $(\sigma, z)$-plane is a particular choice of a general 3D curve dynamics

$$
\boldsymbol{u}=u_{t} \hat{\boldsymbol{t}}+u_{n} \hat{\boldsymbol{n}}+u_{b} \hat{\boldsymbol{b}}
$$

where the tangent $\hat{\boldsymbol{t}}$, normal $\hat{\boldsymbol{n}}$, and binormal $\hat{\boldsymbol{b}} \equiv \hat{\boldsymbol{t}} \times \hat{\boldsymbol{n}}$ comprise the Frenet-Serret triad [12], obeying the equations of differential geometry

$$
\begin{gathered}
\hat{\boldsymbol{t}} \equiv \frac{d \boldsymbol{r}}{d s}, \quad \frac{d \hat{\boldsymbol{t}}}{d s}=\frac{d^{2} \boldsymbol{r}}{d s^{2}}=\kappa \hat{\boldsymbol{n}}, \quad \kappa=\left|\frac{d^{2} \boldsymbol{r}}{d s^{2}}\right| \\
\frac{d \hat{\boldsymbol{b}}}{d s}=-\tau \hat{\boldsymbol{n}}, \quad \tau=\kappa^{-2}\left(\frac{d \boldsymbol{r}}{d s} \times \frac{d^{2} \boldsymbol{r}}{d s^{2}}\right) \cdot \frac{d^{3} \boldsymbol{r}}{d s^{3}} \quad \frac{d \boldsymbol{n}}{d s}=-\kappa \hat{\boldsymbol{t}}+\tau \hat{\boldsymbol{b}}
\end{gathered}
$$

where $\kappa$ is the curvature, $\tau$ is the torsion of the curve at arclength position $s$ and time $t$. The direct evaluation gives

$$
\frac{d s}{d z}=\sqrt{1+\Sigma_{z}^{2}}, \quad \tau=0, \quad \kappa=\frac{\Sigma_{z z}}{1+\Sigma_{z}^{2}}
$$

Axial symmetry of the problem reduces evolution to $(\hat{\boldsymbol{n}}, \hat{\boldsymbol{t}})$-plane and $(z \leftrightarrow s)$ and $(\Sigma(z, t) \leftrightarrow \kappa(s, t))$ equivalence. This describes evolution of an axisymmetric surface pure geometrically in terms of the curvature and the arclength. Integrodifferential form of Eqs. (10 14) leads to strong nonlocality as a consequence of long-range part of the interaction.

\section{SUMMARY}

We consider an uniformly charged incompressible nuclear fluid bounded by a closed surface. It is shown that an evolution of an axisymmetric surface $\Gamma(\boldsymbol{r}, t) \equiv \sigma-\Sigma(z, t)=0, \quad \boldsymbol{r}=(\sigma, \phi, z)$ can be approximately reduced to a motion of a curve in the $(\sigma, z)$-plane. A nonlinear integro-differential equation for the contour $\Sigma(z, t)$ is derived. It is shown the direct correspondence between $\Sigma(z, t)$ and a local curvature, that gives possibility to use methods of differential geometry to analyze an evolution of an axisymmetric nuclear surface.

The goal of this short report is to present the principal line only. The extended paper is in progress.

Work supported in part by Russian Foundation for Basic Research and Deutsche Forschungsgemeinschaft.

[1] J.W.S. Rayleigh, Proc. R. Soc. Lond. 29 (1879) 71.

[2] S. Chandrasekhar, Ellipsoidal Figures of Equilibrium (New Haven: Yale University Press) 1969.

[3] J. M. Eisenberg and W. Greiner, Nuclear Models (Collective and Single-Particle Phenomena), (North Holland Publ. Comp. Amsterdam) 1970

[4] V. G. Kartavenko, Phys. Part. Nucl. 24619 (1993).

[5] R. E. Apfel et al. Phys. Rev. Lett. 78 (1997) 1912.

[6] A. Ludu and J.P. Draayer, Phys. Rev. Lett., 80 (1998) 2125-2128.

[7] V.G. Kartavenko, K.A. Gridnev and W. Greiner, Int. J. Mod. Phys., E7, (1998) 287.

[8] V.G. Kartavenko, A. Săndulescu and W. Greiner, Int. J. Mod. Phys. E, 8 (1999) 381.

[9] V.G. Kartavenko, J. Phys. G: Nucl. Part. Phys. 19 (1993) L83.

[10] V.G. Kartavenko, K.A. Gridnev, J. Maruhn and W. Greiner, J. Phys. G: Nucl. and Part. Phys. 22 (1996) L19

[11] C.E. Rosenkilde, J. Math. Phys. 8 (1967) 84;88;98

[12] V.I. Smirnov, Kurs vyshej mathematiki (in russian) Vol.2, Chapt.V, p.351 GITTL, 1957 